\title{
Neuroprotective Effect of Sodium Butyrate against Cerebral Ischemia/Reperfusion Injury in Mice
}

\author{
Jing Sun, ${ }^{1,2}$ Fangyan Wang, ${ }^{3}$ Haixiao Li, ${ }^{4}$ Huiqing Zhang, ${ }^{4}$ Jiangtao Jin, ${ }^{4}$ Wenqian Chen, \\ Mengqi Pang, ${ }^{4}$ Junjie Yu, ${ }^{4}$ Yiwen He, ${ }^{4}$ Jiaming Liu, ${ }^{4}$ and Chunfeng Liu ${ }^{1}$ \\ ${ }^{1}$ Department of Neurology, The Second Affiliated Hospital of Soochow University, Suzhou, Jiangsu 215004, China \\ ${ }^{2}$ Department of Neurology, The Second Affiliated Hospital of Wenzhou Medical University, Wenzhou, Zhejiang 325027, China \\ ${ }^{3}$ School of Basic Medical Sciences, Wenzhou Medical University, Wenzhou, Zhejiang 325035, China \\ ${ }^{4}$ School of Environmental Science and Public Health, Wenzhou Medical University, Wenzhou, Zhejiang 325035, China
}

Correspondence should be addressed to Jiaming Liu; wzljm@126.com and Chunfeng Liu; liucf@suda.edu.cn

Received 12 February 2015; Accepted 24 April 2015

Academic Editor: Antoni Camins

Copyright (c) 2015 Jing Sun et al. This is an open access article distributed under the Creative Commons Attribution License, which permits unrestricted use, distribution, and reproduction in any medium, provided the original work is properly cited.

\begin{abstract}
Sodium butyrate $(\mathrm{NaB})$ is a dietary microbial fermentation product of fiber and serves as an important neuromodulator in the central nervous system. In this study, we further investigated that $\mathrm{NaB}$ attenuated cerebral ischemia/reperfusion (I/R) injury in vivo and its possible mechanisms. $\mathrm{NaB}(5,10 \mathrm{mg} / \mathrm{kg})$ was administered intragastrically $3 \mathrm{~h}$ after the onset of reperfusion in bilateral common carotid artery occlusion (BCCAO) mice. After $24 \mathrm{~h}$ of reperfusion, neurological deficits scores were estimated. Morphological examination was performed by electron microscopy and hematoxylin-eosin (H\&E) staining. The levels of oxidative stress and inflammatory cytokines were assessed. Apoptotic neurons were measured by TUNEL; apoptosis-related protein caspase3, Bcl-2, Bax, the phosphorylation Akt (p-Akt), and BDNF were assayed by western blot and immunohistochemistry. The results showed that $10 \mathrm{mg} / \mathrm{kg} \mathrm{NaB}$ treatment significantly ameliorated neurological deficit and histopathology changes in cerebral I/R injury. Moreover, $10 \mathrm{mg} / \mathrm{kg} \mathrm{NaB}$ treatment markedly restored the levels of MDA, SOD, IL- $1 \beta$, TNF- $\alpha$, and IL-8. $10 \mathrm{mg} / \mathrm{kg}$ NaB treatment also remarkably inhibited the apoptosis, decreasing the levels of caspase- 3 and Bax and increasing the levels of Bcl2, p-Akt, and BDNF. This study suggested that NaB exerts neuroprotective effects on cerebral I/R injury by antioxidant, antiinflammatory, and antiapoptotic properties and BDNF-PI3K/Akt pathway is involved in antiapoptotic effect.
\end{abstract}

\section{Introduction}

Accumulating evidence has demonstrated that cerebral ischemia/reperfusion ( $I / R)$ injury often causes irreversible brain damage and the cascade of events causing neuronal injury and death, which is involved in many complex factors such as deprivation of blood flow, oxidative stress, inflammation, and apoptosis [1,2]. The cerebral I/R injury can result in functional impairment and/or neuronal death [3]. Oxidative stress is one of the primary factors that can aggravate the cerebral I/R injury. Excessive production of reactive oxygen species (ROS) may directly contribute to destruction of the cell membrane by inducing lipid peroxidation [4]. In addition, antioxidant is an effective substance, which has been shown to alleviate neuronal damage from brain ischemia injury [5]. Furthermore, significant inflammatory response has been observed during ischemic stroke [2]. Numbers data has indicated that various inflammatory mediators such as IL-1 $\beta$, IL-8, and TNF- $\alpha$ can lead to neuronal damage $[6,7]$. Apoptosis is generally considered to play a major role in delayed neuronal death following cerebral I/R [8]. Several studies have shown that many signal pathways could be involved in antiapoptosis and phosphoinositide 3-kinase/protein kinase B (PI3K/Akt) signaling pathway is essential for the cell growth and survival in brain I/R injury [9]. Taking into account the fact that cerebral I/R injury is associated with the oxidative, inflammatory, and apoptotic mechanism, some are is willing to explore the neuroprotective agents that may attenuate the cerebral $\mathrm{I} / \mathrm{R}$ damage. At present, natural products, especially microbial fermentation products, probably represent an ideal source to develop safe and effective agents for management of cerebral I/R injury. 
Sodium butyrate $(\mathrm{NaB})$, a dietary microbial fermentation product of fiber, is a sort of short chain fatty acids (SCFAs), which has been previously shown to inhibit intestinal pathogenic bacteria and maintain gastrointestinal homeostasis [10]. Recent studies have indicated that $\mathrm{NaB}$ could provide anti-inflammatory and neuroprotective effects in neurodegenerative disorders [11] and also improve spatial learning and memory ability [12], myocardial I/R injury [13], acute lung injury [14], and acute liver failure [15]. Although the neuroprotective effect of $\mathrm{NaB}$ has been well studied, its neuroprotective effects on the cerebral I/R injury have not yet been clear. Therefore, the present study was performed to investigate the neuroprotective effect and possible mechanisms of $\mathrm{NaB}$ treatment in cerebral $\mathrm{I} / \mathrm{R}$ injury induced by the bilateral common carotid arteries occlusion (BCCAO) in mice.

\section{Materials and Methods}

2.1. Animals. All procedures involving animals were approved by and conformed to the guidelines of the Institutional Animal Care Committee of Wenzhou Medical University. Male ICR mice (6-week old, 22-24 g) were obtained from Experiment Animal Center of Wenzhou Medical University. The mice were housed in a pathogen-free animal facility and maintained on a $12 \mathrm{~h}$ light/dark cycle. Food and water were available ad libitum [16].

2.2. Surgical Procedures of Cerebral I/R Injury Mice Model. Cerebral I/R injury mice model was induced by the bilateral common carotid arteries occlusion (BCCAO) with vascular clips $[17,18]$. The mice were anaesthetized with $400 \mathrm{mg} / \mathrm{kg}$ injection of chloral hydrate and a midline incision in the ventral side of the neck was made to expose the right and left common carotid arteries. We gently separated the two arteries and occluded them with vascular clips for $20 \mathrm{~min}$, and then the clips were removed to restore blood for recirculation. The sham-operated mice underwent the same operation procedure, but the common carotid arteries were not occluded $[19,20]$.

2.3. Drug Administration and Groups. $\mathrm{NaB}$ (sodium butyrate, sigma Co. Ltd., USA with a purity $\geq 99 \%$ ) was dissolved in normal saline and intragastrically administered $3 \mathrm{~h}$ after the onset of reperfusion in mice. The mice were randomly divided into four groups $(n=12)$ as follows: (1) sham-operated group; (2) cerebral I/R model group; (3-4) $\mathrm{NaB}$ treatment groups, which were subjected to I/R model and treated with $\mathrm{NaB} 5$ and $10 \mathrm{mg} / \mathrm{kg}$, respectively. Shamoperated and model groups were given an identical dose of normal saline to serve as a control.

2.4. Neurological Function Assessment. Evaluation of the neurological deficits at $24 \mathrm{~h}$ after the reperfusion was based on the method of Longa [13]. Neurological function was scored on a five-point scale: 0 , no deficits; 1 , difficulty in fully extending the contralateral forelimb; 2 , not being able to extend the contralateral forelimb; 3 , mild circling to the contralateral side; 4, severe circling; and 5, falling to the contralateral side. The higher the neurological deficit score is, the severer the injury is. Ten mice were evaluated in each group.

2.5. Morphological Evaluation. After $24 \mathrm{~h}$ of reperfusion, brain tissues were rapidly removed and fixed with $25 \%$ glutaraldehyde for $2 \mathrm{~h}$ at $4^{\circ} \mathrm{C}$, rinsed with PBS, and soaked in osmium tetroxide. Ultrathin sections were prepared and placed onto colloid coated copper grids and double-stained with $0.4 \%$ uranyl acetate and $2 \%$ lead acetate. The ultrastructure of the neurons was observed by electron microscopy.

The brains were processed routinely for paraffin embedding and sectioning into $5 \mu \mathrm{m}$ thickness, and the sections were stained with hematoxylin-eosin ( $\mathrm{H} \& \mathrm{E})$ according to the methods described previously [21]. The normal tissue was stained to deep red-blue and the ischemia area was stained to pale pink. The slides were photographed under light microscope.

2.6. Biochemical Determination. Brain tissues were weighed and homogenized for MDA, SOD activity, IL- $1 \beta$, IL- 8 , and TNF- $\alpha$ assay in conformance with ELISA kits. The content of MDA and enzymatic activities of SOD were measured using ELISA kits (Nanjing Jiancheng Bioengineering Institute), and the contents of IL- $1 \beta$, IL- 8 , and TNF- $\alpha$ were measured using ELISA kits (RayBiotech, Norcross, GA, USA).

2.7. TUNEL Staining. Paraffin-embedded brains were sectioned in $5 \mu \mathrm{m}$ thickness and stained with TUNEL assay kit for apoptotic cells, according to the previous methods [16]. The positive cells displayed a brown stain within the cytoplasm of the apoptotic cells [22]. The slides were photographed (400x magnification).

2.8. Western Blotting. A total of $20 \mu \mathrm{g}$ protein samples, obtained from the ischemic brain tissues, were loaded onto a $12 \%$ SDS-PAGE gel. After the electrophoresis, proteins were transferred to a nitrocellulose membrane. Nonspecific binding sites were blocked with 5\% nonfat milk in TBST for $1 \mathrm{~h}$ and then incubated with primary antibodies against BDNF, Akt, p-Akt, Bcl-2, Bax, caspase-3, and $\beta$-actin (1:1000, Bioworld, USA) overnight at $4^{\circ} \mathrm{C}$. Subsequently, blots were incubated with HRP-conjugated secondary antibody at room temperature for $1 \mathrm{~h}$. The protein bands were visualized with ECL chemiluminescence system (Thermo Scientific, Rockford, IL). The optical density was quantified by performing the National Institute of Health ImageJ software. $\beta$-actin was performed as a loading control.

2.9. Immunohistochemistry. Immunohistochemistry for Bcl2 and Bax was carried out on paraffin-embedded sections. The primary antibody of Bcl-2 (1:100, Cell Signaling Technology, USA) and Bax (1:250, Bioworld, USA) was applied overnight at $4^{\circ} \mathrm{C}$. The sections were washed and then incubated with secondary antibodies (goat-anti-rabbit, 1:500), and the sections were visualized using diaminobenzidine $(\mathrm{DAB})$ as the chromogen. Brown granules in cells under 


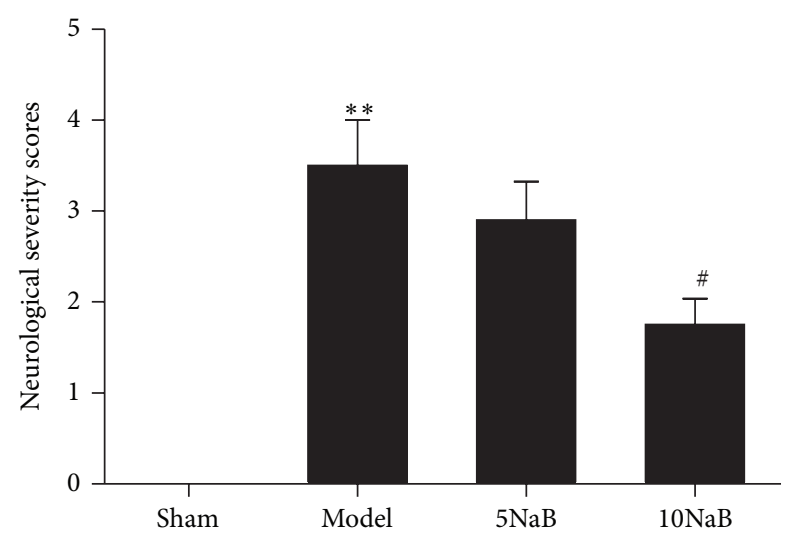

Figure 1: Effect of $\mathrm{NaB}$ on neurological deficit scores. ${ }^{* *} p<0.01$ versus sham group and ${ }^{\#} p<0.05$ versus model group. $n=10$, each group.

microscope were defined as positive signals and observed at 400x magnification of light microscopy.

2.10. Statistical Analysis. The data were expressed as mean \pm SD and analyzed using the SPSS 17.0 statistical analytical software (SPSS, Chicago, IL, USA). Statistical analysis of the results was carried out by one-way ANOVA, followed by fisher post hoc comparisons. Statistical significance was assumed for $p<0.05$.

\section{Results}

3.1. NaB Improved Neurological Deficit. The ischemia of the cerebral hippocampus caused a deficit in neurological function in the mice that was mainly visible as left forelimb paralysis. In this study, we examined the neuroprotective effect of $\mathrm{NaB}$ treatment using neurological deficit scores. As shown in Figure 1, all mice except sham-operated ones presented neurological deficit $(p>0.05)$. The mice in $10 \mathrm{mg} / \mathrm{kg} \mathrm{NaB}$ treatment groups exhibited significantly improved neurological deficits scores after the cerebral I/R injury compared with the model group $(p<0.01)$. These results suggested that $10 \mathrm{mg} / \mathrm{kg} \mathrm{NaB}$ treatment effectively attenuated the neurological dysfunction of cerebral $I / R$ in mice.

3.2. NaB Protects against Histological Changes. As shown in Figure 2, no destructive changes were observed in the sham group by electron microscopy; the normal neurons contained large oval nucleuses and clear mitochondria. The irregular nucleuses of neurons and swollen mitochondria were observed in the model mice. By contrast, $\mathrm{NaB}$ attenuated the impairment of the ultrastructural neurons in the cerebral I/R injury mice.

The histopathological abnormalities in the hippocampal CA1 region were measured by the $\mathrm{H} \& \mathrm{E}$ staining. As illustrated in Figure 3, shrunken and pycnotic nuclei of CA1 neurons were exhibited in the model group. By contrast, these neuronal damages were dramatically reduced by the $\mathrm{NaB}$ treatments.

3.3. NaB Attenuates the Oxidative Stress. To investigate the effects of $\mathrm{NaB}$ on lipid peroxidation and free radical activity induced by BCCAO, the contents of MDA and the activities of SOD in the brain tissues were measured. After $24 \mathrm{~h}$ of reperfusion, the activities of SOD in ischemic hippocampus of the model group were diminished remarkably compared with the sham group $(p<0.01$, Figure 4(a)). However, the SOD activity in the $10 \mathrm{mg} / \mathrm{kg} \mathrm{NaB}$ treatment group was significantly enhanced compared with the model group $(p<$ 0.05 , Figure 4(a)). The content of MDA was also increased in the model group compared with the sham group $(p<0.01$, Figure 4(b)). After $10 \mathrm{mg} / \mathrm{kg} \mathrm{NaB}$ treatment, the content of MDA was evidently deceased in comparison to the model group $(p<0.05$, Figure $4(\mathrm{~b}))$. These results suggested that $\mathrm{NaB}(10 \mathrm{mg} / \mathrm{kg})$ treatment effectively attenuated the brain oxidative stress of the cerebral I/R in mice.

3.4. NaB Attenuates the Inflammatory Response. Inflammation is one of the key pathogenic events in the cerebral I/R injury. We examined the cytokines (IL-1 $\beta$, IL-8, and TNF$\alpha$ ) in the ischemic hippocampus after $24 \mathrm{~h}$ of reperfusion by ELISA. As shown in Figure 5, levels of IL-1 $\beta$ ( $p<0.01)$, IL-8 $(p<0.05)$, and TNF- $\alpha(p<0.01)$ were markedly increased in the model group relative to the sham group. However, $10 \mathrm{mg} / \mathrm{kg} \mathrm{NaB}$ treatment group significantly decreased the levels of these cytokines relative to the model group $(p<$ $0.05)$, especially the level of IL-1 $\beta(p<0.01)$.

3.5. NaB Attenuates the Apoptosis on the Hippocampus. TUNEL assay was used to exam the typical DNA laddering pattern of neurons in the ischemic penumbra regions after cerebral I/R injury. As shown in Figure 6(a), we found that the number of apoptotic cells in the hippocampus region $24 \mathrm{~h}$ after BCCAO and reperfusion was increased significantly compared with the sham group. However, the number of apoptotic cells was decreased significantly in the $10 \mathrm{mg} / \mathrm{kg}$ $\mathrm{NaB}$ treatment group compared with model group, suggesting the antiapoptotic activity of $\mathrm{NaB}$ on the hippocampus.

3.6. NaB Activated BDNF-PI3K/Akt Pathway-Related Proteins (Caspase-3, Bcl-2, Bax, Akt, p-Akt, and BDNF) Assessed by Western Blot and Immunohistochemistry. In order to evaluate the protective effect of $\mathrm{NaB}$ on BDNF-PI3K/Akt pathway after cerebral I/R injury, we examined the protein levels of caspase-3, Bcl-2, Bax, Akt, p-Akt (ser473), and BDNF by western blot analysis. The protein level of caspase- 3 in the model group was abundantly increased compared with the sham group, whereas caspase-3 in $10 \mathrm{mg} / \mathrm{kg} \mathrm{NaB}$ treatment group was dramatically decreased compared with the model group (Figures 7(a) and 7(e)). The $10 \mathrm{mg} / \mathrm{kg} \mathrm{NaB}$ treatment markedly increased the levels of Bcl-2 and decreased the levels of Bax in mice after cerebral I/R injury (Figure 7(a)). The ratio of $\mathrm{Bcl}-2 / \mathrm{Bax}$ (antiapoptotic/proapoptotic) was significantly reduced in the model group compared with the sham group $(p<0.01$, Figure $7(\mathrm{~d}))$. However, 

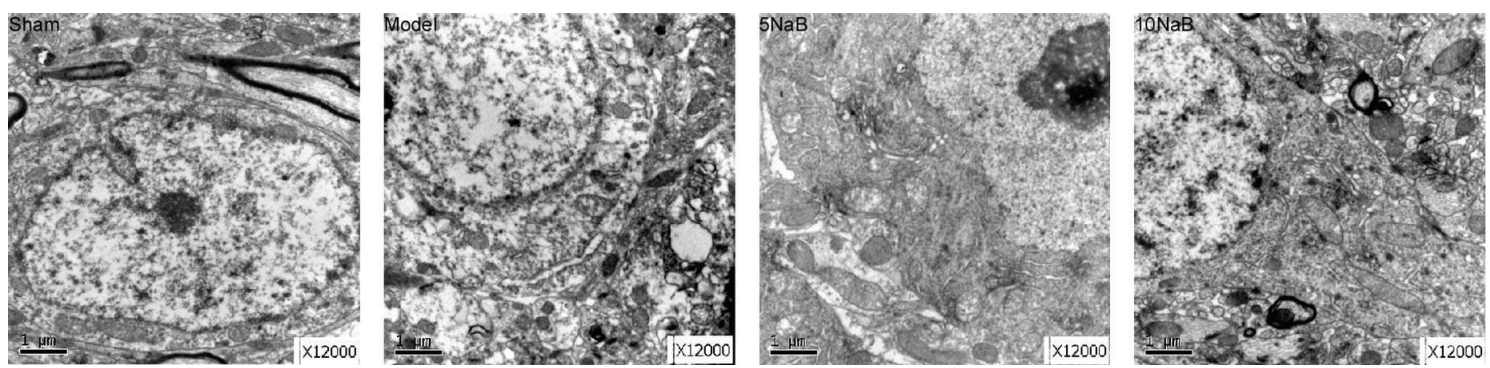

FIGURE 2: Effect of $\mathrm{NaB}$ on ultrastructure. Representative photomicrographs of ultrastructure; magnification: 12,000x. Scale bar $=1 \mu \mathrm{m}$.
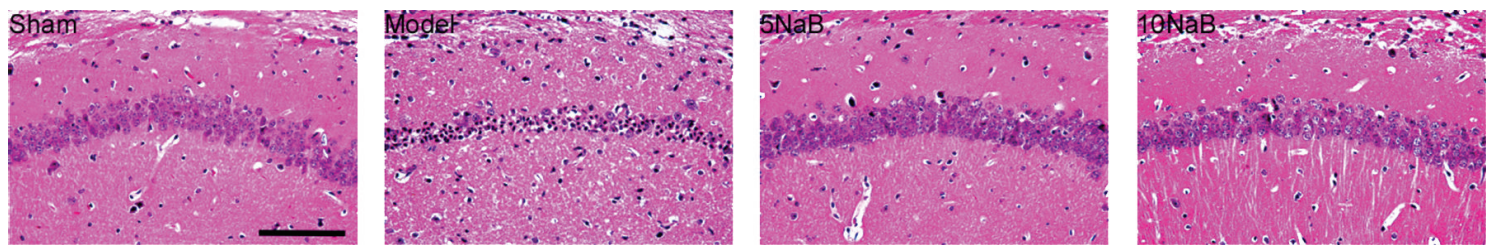

FIGURE 3: Effect of $\mathrm{NaB}$ on histopathology. Representative photomicrographs of H\&E staining. Cells with brown stained cytoplasm are positive cells. Magnification: 400x. Scale bar $=20 \mu \mathrm{m}$.

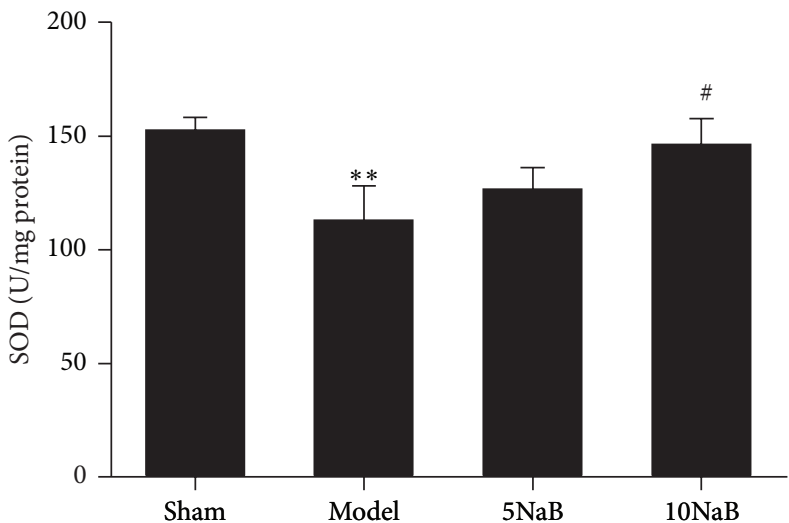

(a)

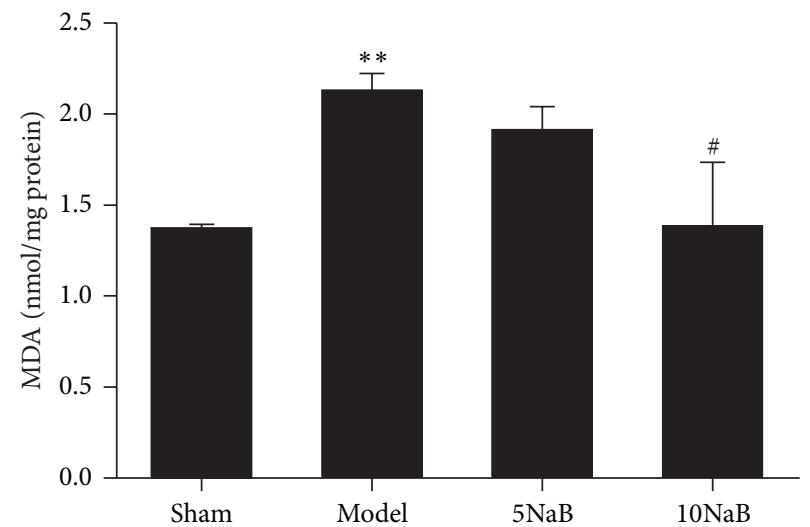

(b)

Figure 4: Effect of $\mathrm{NaB}$ on the levels of SOD and MDA. SOD assay (a) and MDA assay (b). ${ }^{* *} p<0.01$ versus sham group and ${ }^{\#} p<0.05$ versus model group. $n=6$, each group.

the ratio of $\mathrm{Bcl}-2 / \mathrm{Bax}$ in $10 \mathrm{mg} / \mathrm{kg} \mathrm{NaB}$ treatment group was remarkably increased compared with the model group $(p<0.01$, Figure $7(\mathrm{~d}))$. There was no significant difference in protein level of Akt among the four groups $(p>0.01$, Figure 7(a)). However, the level of p-Akt in the model group was significantly reduced in comparison to the sham group, increased in $10 \mathrm{mg} / \mathrm{kg} \mathrm{NaB}$ treatment group (Figure 7(a)). The ratio of p-Akt/Akt was significantly descended in the model group in comparison to the sham group $(p<0.05$, Figure $7(\mathrm{c})$ ). However, the ratio in $10 \mathrm{mg} / \mathrm{kg} \mathrm{NaB}$ treatment group was significantly increased compared with the model group ( $p<0.01$, Figure $7(\mathrm{c}))$. The protein level of BDNF in the model group was significantly decreased compared with the sham group, whereas the $10 \mathrm{mg} / \mathrm{kg} \mathrm{NaB}$ treatment group was significantly increased compared with the model group $(p<0.01$, Figures 7(a) and 7(b)). There was no significant difference between the $5 \mathrm{mg} / \mathrm{kg} \mathrm{NaB}$ treatment group and the model group $(p>0.05$, Figure 7$)$. These findings implied that $\mathrm{NaB}$ treatment could activate BDNF-PI3K/Akt pathway.

The above results were also partly confirmed by immunohistochemistry. As shown in Figures 6(b) and 6(c), few positive cells of Bax while plenty of positive cells of Bcl-2 could be found in the sham group. In the model group, the positive cells of Bax were numerous, while Bcl-2 was less compared with the sham group in the hippocampal CA1 region. After the $\mathrm{NaB}$ treatment, the number of the positive cells of Bax was significantly decreased, whereas the number of the positive cells of Bcl2 was significantly increased in comparison to the model group. 


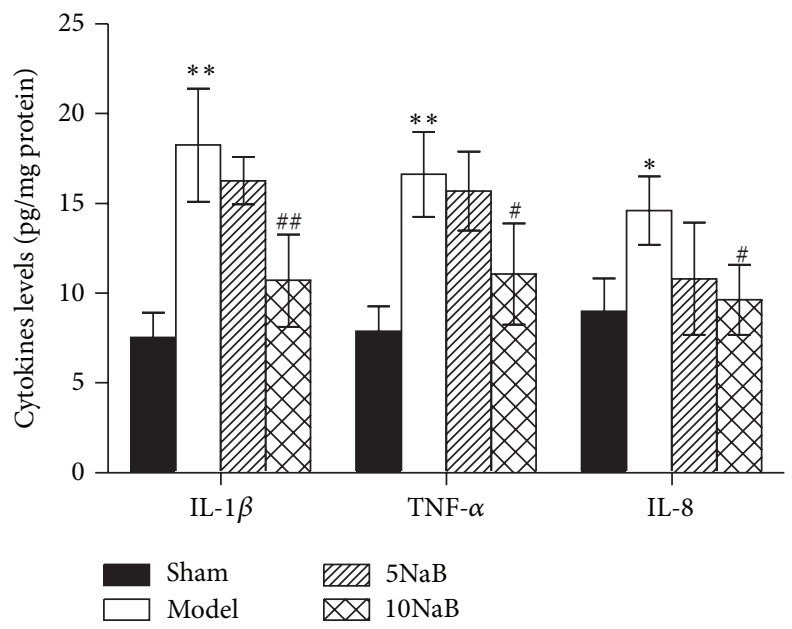

Figure 5: Effect of $\mathrm{NaB}$ on the levels of IL-1 $\beta$, IL-8, and TNF- $\alpha .{ }^{*} p<0.05$ versus sham group, ${ }^{* *} p<0.01$ versus sham group, ${ }^{\#} p<0.05$ versus model group, and ${ }^{\# \#} p<0.05$ versus model group. $n=6$, each group.
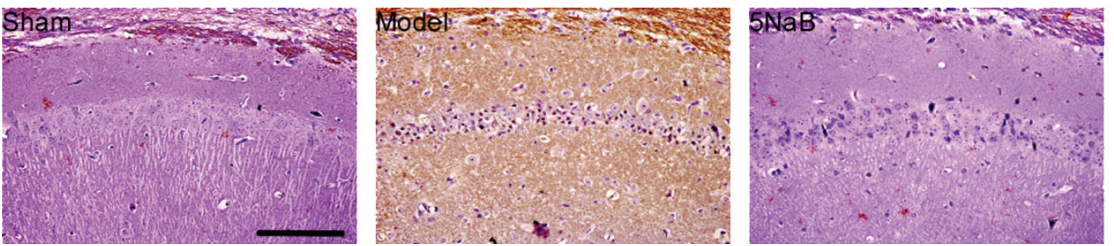

(a)
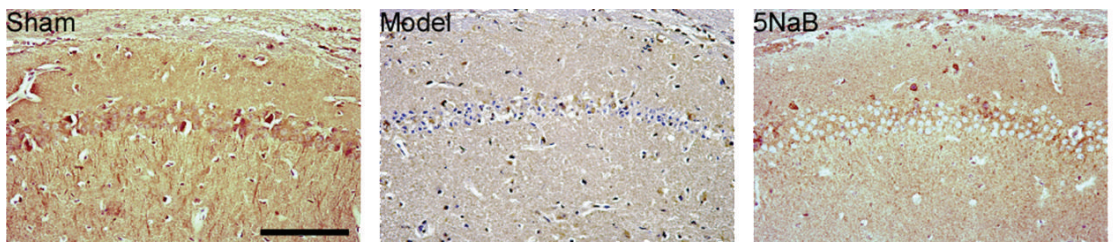

(b)
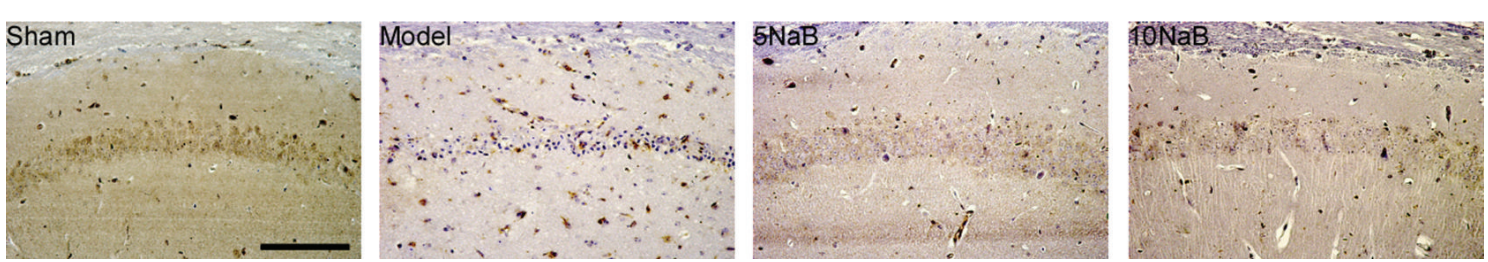

(c)
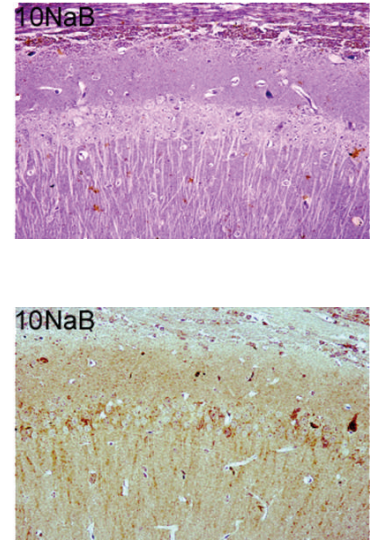

Figure 6: $\mathrm{NaB}$ attenuates the apoptosis on the hippocampus. Representative photomicrographs of TUNEL staining (a), active Bcl-2 immunohistochemistry (b), and active Bax immunohistochemistry (c). The cells with the brown-stained cytoplasm were the positive cells. Magnification: 400x. Scale bar $=20 \mu \mathrm{m}$.

\section{Discussion}

Although many mechanisms are involved in the pathogenesis of stoke, numerous studies show that oxidative stress, inflammation, and apoptosis mainly account for its pathogenic progression $[22,23]$. The results of this study demonstrated that $\mathrm{NaB}$ may protect against cerebral I/R injury by improving neurological dysfunction and pathological changes, restoring the levels of MDA and SOD and decreasing the levels of proinflammatory cytokines IL- $1 \beta$, IL-8, and TNF- $\alpha$ after cerebral I/R injury. The underlying mechanism of this antiapoptotic effect may be involved in the activation of the BNDF-PI3K/Akt pathway.

$\mathrm{NaB}$, a dietary microbial fermentation product of fiber, possesses multiple bioactivities. Recent findings have shown that it could effectively inhibit inflammation and neuronal apoptosis in the animal of brain disorders [11]. Furthermore, it has markedly improved associative and spatial learning and memory in the mice of $\mathrm{AD}$ [24]. However, the protective role of $\mathrm{NaB}$ in cerebral I/R injury has not been clear. 


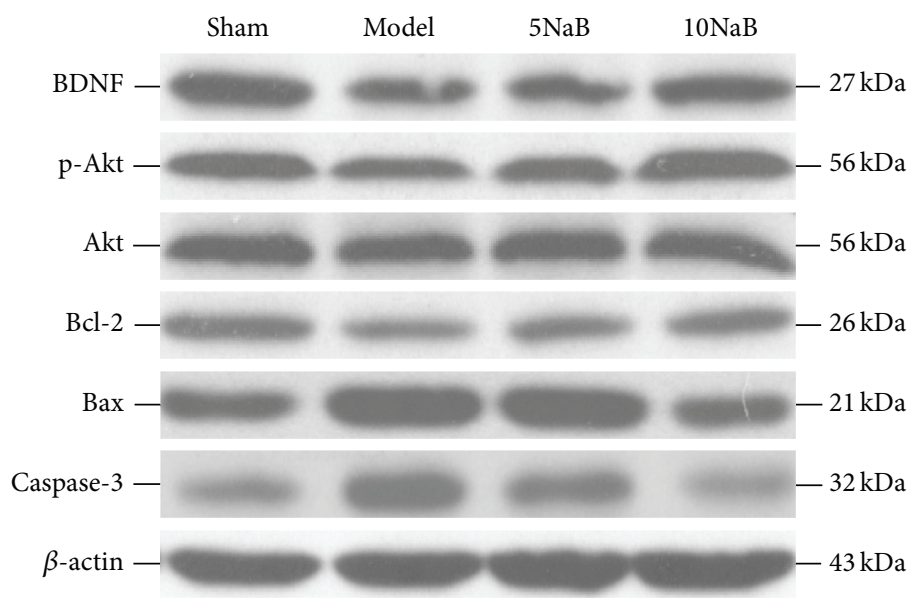

(a)

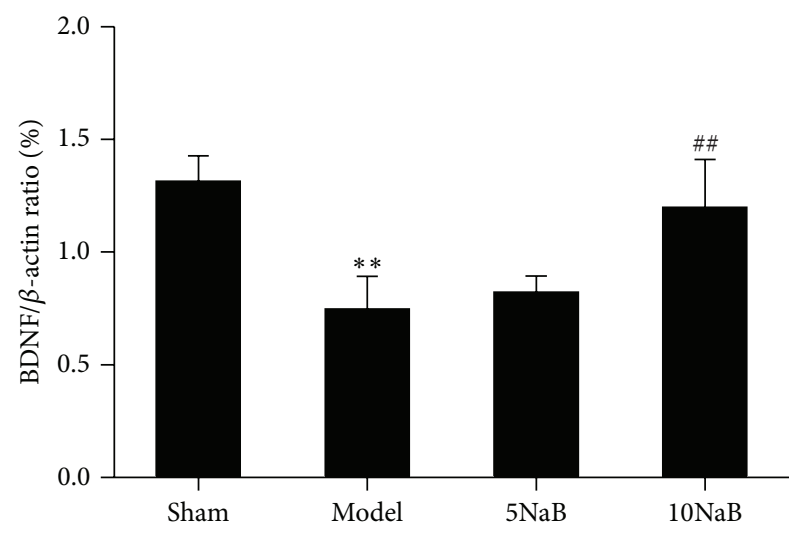

(b)

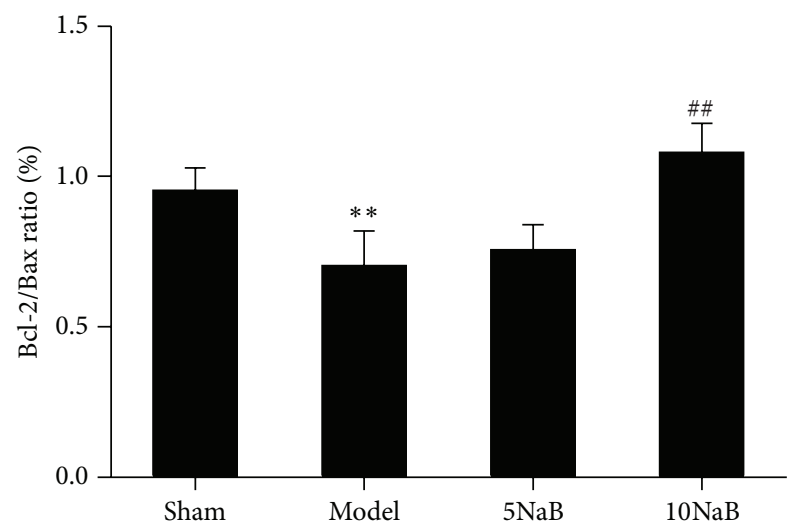

(d)

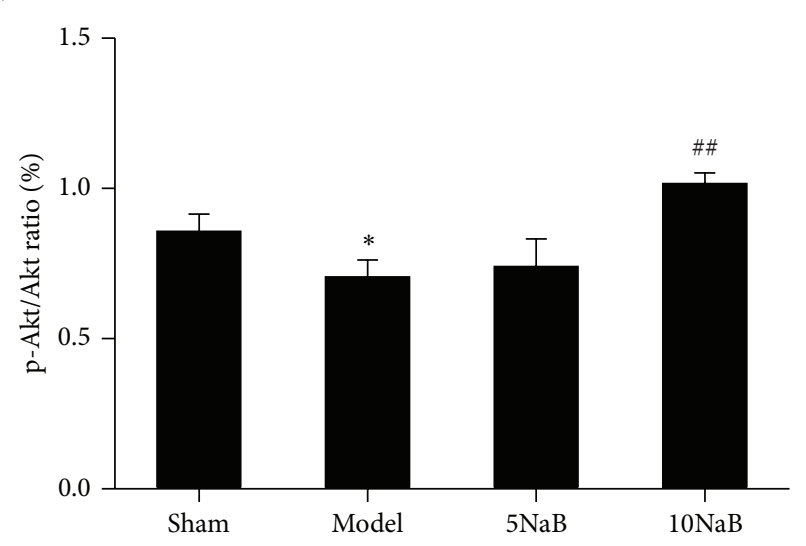

(c)

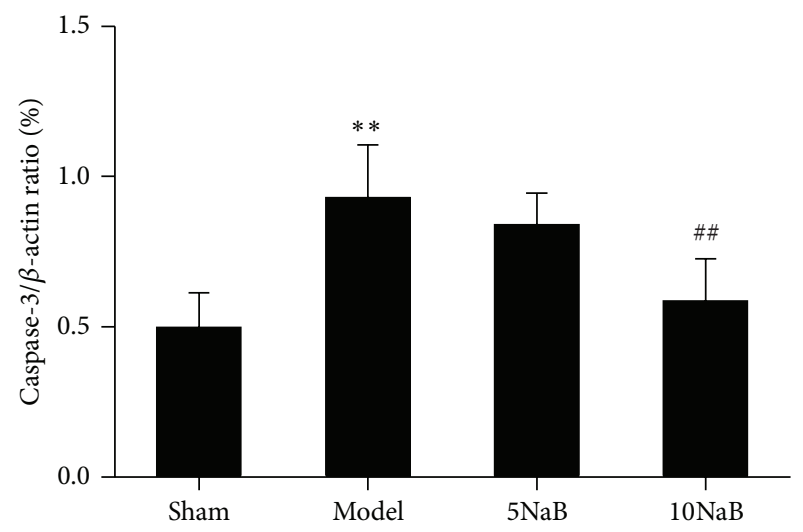

(e)

Figure 7: Effect of $\mathrm{NaB}$ on western blot of apoptosis-related protein (caspase-3, Bcl-2, Bax, Akt, p-Akt, and BDNF). Western blot studies of BDNF, p-Akt, Akt, Bcl-2, Bax, and caspase-3 (a) and the ratios of the protein levels of the BDNF/ $\beta$-actin, p-Akt/Akt, Bcl-2/Bax, and caspase$3 / \beta$-actin (b), (c), (d), and (e). ${ }^{*} p<0.05$ versus sham group, ${ }^{* *} p<0.01$ versus sham group, ${ }^{\#} p<0.05$ versus model group, and ${ }^{\# \#} p<0.05$ versus model group. $n=6$, each group.

In order to develop cerebral I/R injury in mice, we well established cerebral $\mathrm{I} / \mathrm{R}$ mice model induced by BCCAO $[17,18]$, which resulted in morphological and functional changes such as extensive death of hippocampal CA1 pyramidal neurons. The reports show a very wide range of effective doses of $\mathrm{NaB}$ [25-28], and these varieties in dosage might be related to the different animal species and models. According to the preliminary experiment, we chose the dosage of 5 and $10 \mathrm{mg} / \mathrm{kg}$ for $\mathrm{NaB}$. Our results indicated that the $\mathrm{NaB}$ treatment effectively prevented neuropathological 
alterations in hippocampus thereby resulting in improvement of neurological deficit in mice after the cerebral I/R injury induced by BCCAO.

The brain is very sensitive to ROS-mediated injury. Numerous studies have shown that ROS are produced in the brain during I/R injury and directly involved in oxidative damage in ischemic tissue, which led to cell death [29]. SOD is an endogenous antioxidant, which plays a role in the prevention of oxidative injury. Enhancement of antioxidant activities in brain tissues is beneficial for alleviating neuronal damage from cerebral I/R [5, 30, 31]. Recent studies have indicated that $\mathrm{NaB}$ possesses an antioxidant feature in the intestinal epithelial cells [32]. However, its antioxidant effects on cerebral I/R have not yet been reported in mice. We found that $10 \mathrm{mg} / \mathrm{kg} \mathrm{NaB}$ treatment markedly restored the levels of MDA and SOD after the cerebral I/R injury.

Inflammatory reaction plays a vital role in aggravating brain damage during acute stoke [6]. After the occlusion of cerebral blood flow, tissue damage was accompanied by the inflammatory reaction. Cytokines such as IL- $\beta$, IL8 , and TNF- $\alpha$, as mediators for regulating the innate and adaptive immune systems, are changed in the brain with various diseases including ischemic brain injury $[2,7]$. In the present study, levels of IL- $1 \beta$, IL- 8 , and TNF- $\alpha$ were markedly increased in the model group relative to the sham group. Consistently, this study found that $10 \mathrm{mg} / \mathrm{kg} \mathrm{NaB}$ treatment alleviated cerebral $\mathrm{I} / \mathrm{R}$ injury by lessening inflammatory factors IL- $1 \beta$, IL- 8 , and TNF- $\alpha$. The fact that NaB could provide anti-inflammatory effects in acute lung injury has been reported [15].

Oxidative stress and inflammation can also cause neuronal apoptosis. Apoptosis plays an important role in the process of neuronal death after cerebral ischemia [33, 34]. Recent studies have reported that $\mathrm{NaB}$ exerted neuroprotective effects by inhibiting neuronal apoptosis [31]. As a result of $\mathrm{NaB}$ treatment, a marked reduction in the number of TUNEL-positive cells in the hippocampus was observed.

It has been reported that BDNF could exert neuroprotective effect against ischemic brain injury in vivo via activating PI3K/Akt signaling pathway [35]. Akt, downstream of PI3K, is considered to be one of the important factors in cell growth and survival. It is well known that $\mathrm{Bcl}-2, \mathrm{Bax}$, and caspase3 are the downstream target of Akt [36]. Akt activation suppresses apoptosis and promotes cell survival by phosphorylation and subsequent inactivation of apoptosis-inducing factors. Bcl-2 is an antiapoptotic factor and important for cell survival while Bax promotes apoptosis. Caspase-3, a key factor in apoptosis, eventually leads to DNA fragmentation and triggers apoptosis in the acute stage of stroke [23, 37]. Activation of PI3K/Akt pathway has been widely reported to participate in the protection against cerebral I/R injury [38]. In the present study, our result showed that $10 \mathrm{mg} / \mathrm{kg}$ $\mathrm{NaB}$ treatment significantly enhanced the phosphorylation of Akt. Simultaneously, $10 \mathrm{mg} / \mathrm{kg} \mathrm{NaB}$ treatment markedly increased the levels of BDNF, p-Akt, and Bcl-2 and decreased the levels of Bax and caspases-3 in mice after cerebral I/R injury. Taken together, these results indicated that BDNFPI3K/Akt pathway might be involved in antiapoptotic effect of $\mathrm{NaB}$ on cerebral I/R injury in mice.

\section{Conclusion}

In summary, this study firstly demonstrated that $\mathrm{NaB}$ attenuated cerebral I/R injury by minimizing oxidative stress and inflammatory and reducing apoptosis in vivo. Meanwhile, $\mathrm{NaB}$ exerted its antiapoptotic properties in cerebral I/R injury via BDNF-PI3K/Akt signaling pathway and showed a therapeutic potential for treatment of stoke in clinic.

\section{Conflict of Interests}

The authors declare that there is no conflict of interests regarding the publication of this paper.

\section{Authors' Contribution}

Jing Sun and Fangyan Wang equally contributed to this work.

\section{Acknowledgments}

The research was supported by Zhejiang Science and Technology Development Funds (2013C37005) and the Project supported by Zhejiang Provincial Natural Science Foundation of China (LY13B070012).

\section{References}

[1] G. H. Danton and W. D. Dietrich, "Inflammatory mechanisms after ischemia and stroke," Journal of Neuropathology and Experimental Neurology, vol. 62, no. 2, pp. 127-136, 2003.

[2] T. J. Kleinig and R. Vink, "Suppression of inflammation in ischemic and hemorrhagic stroke: therapeutic options," Current Opinion in Neurology, vol. 22, no. 3, pp. 294-301, 2009.

[3] M. Ashafaq, M. M. Khan, S. Shadab Raza et al., "S-allyl cysteine mitigates oxidative damage and improves neurologic deficit in a rat model of focal cerebral ischemia," Nutrition Research, vol. 32, no. 2, pp. 133-143, 2012.

[4] P. H. Chan, "Mitochondria and neuronal death/survival signaling pathways in cerebral ischemia," Neurochemical Research, vol. 29, no. 11, pp. 1943-1949, 2004.

[5] P. H. Chan, "Role of oxidants in ischemic brain damage," Stroke, vol. 27, no. 6, pp. 1124-1129, 1996.

[6] G. Pignataro, A. Scorziello, G. Di Renzo, and L. Annunziato, "Post-ischemic brain damage: effect of ischemic preconditioning and postconditioning and identification of potential candidates for stroke therapy," The FEBS Journal, vol. 276, no. 1, pp. 46-57, 2009.

[7] W. Xia, J. Han, G. Huang, and W. Ying, "Inflammation in ischaemic brain injury: current advances and future perspectives," Clinical and Experimental Pharmacology \& Physiology, vol. 37, no. 2, pp. 253-258, 2010.

[8] R. Brouns and P. P. de Deyn, "The complexity of neurobiological processes in acute ischemic stroke," Clinical Neurology and Neurosurgery, vol. 111, no. 6, pp. 483-495, 2009.

[9] S. K. Mahajan, R. Kashyap, B. R. Sood et al., "Stroke at moderate altitude," The Journal of Association of Physicians of India, vol. 52, pp. 699-702, 2004.

[10] T. Liu, J. Li, Y. Liu et al., "Short-chain fatty acids suppress lipopolysaccharide-induced production of nitric oxide and proinflammatory cytokines through inhibition of NF-kappaB 
pathway in RAW264.7 cells," Inflammation, vol. 35, no. 5, pp. 1676-1684, 2012.

[11] D.-M. Chuang, Y. Leng, Z. Marinova, H.-J. Kim, and C.-T. Chiu, "Multiple roles of HDAC inhibition in neurodegenerative conditions," Trends in Neurosciences, vol. 32, no. 11, pp. 591-601, 2009.

[12] A. Fischer, F. Sananbenesi, X. Wang, M. Dobbin, and L.-H. Tsai, "Recovery of learning and memory is associated with chromatin remodelling," Nature, vol. 447, no. 7141, pp. 178-182, 2007.

[13] X. R. Hu, K. Zhang, C. W. Xu, Z. Q. Chen, and H. Jiang, "Anti-inflammatory effect of sodium butyrate preconditioning during myocardial ischemia/reperfusion," Experimental and Therapeutic Medicine, vol. 8, no. 1, pp. 229-232, 2014.

[14] X. Liang, R.-S. Wang, F. Wang et al., "Sodium butyrate protects against severe burn-induced remote acute lung injury in rats," PLoS ONE, vol. 8, no. 7, Article ID e68786, 2013.

[15] F. Yang, L. K. Wang, X. Li, L. W. Wang, X. Q. Han, and Z. J. Gong, "Sodium butyrate protects against toxin-induced acute liver failure in rats", Hepatobiliary \& Pancreatic Diseases International, vol. 13, no. 3, pp. 309-315, 2014.

[16] J. Zhu, Y. Jiang, L. Wu, T. Lu, G. Xu, and X. Liu, "Suppression of local inflammation contributes to the neuroprotective effect of ginsenoside Rb1 in rats with cerebral ischemia," Neuroscience, vol. 202, pp. 342-351, 2012.

[17] M. A. Moskowitz, K. J. Kiwak, K. Hekimian, and L. Levine, "Synthesis of compounds with properties of leukotrienes C4 and D4 in gerbil brains after ischemia and reperfusion," Science, vol. 224, no. 4651, pp. 886-889, 1984.

[18] X. Zhao, R. Strong, J. Zhang et al., "Neuronal PPAR $\gamma$ deficiency increases susceptibility to brain damage after cerebral ischemia," The Journal of Neuroscience, vol. 29, no. 19, pp. 6186-6195, 2009.

[19] R. Cipriani, P. Villa, G. Chece et al., "CX3CL1 is neuroprotective in permanent focal cerebral ischemia in rodents," The Journal of Neuroscience, vol. 31, no. 45, pp. 16327-16335, 2011.

[20] X. Zhai, X. Chen, J. Shi et al., "Lactulose ameliorates cerebral ischemia-reperfusion injury in ratsby inducing hydrogen by activating Nrf2 expression," Free Radical Biology and Medicine, vol. 65, pp. 731-741, 2013.

[21] Y. Cao, X. Mao, C. Sun et al., "Baicalin attenuates global cerebral ischemia/reperfusion injury in gerbils via anti-oxidative and anti-apoptotic pathways," Brain Research Bulletin, vol. 85, no. 6, pp. 396-402, 2011.

[22] J. Li, Y. Qu, D. Chen et al., "The neuroprotective role and mechanisms of TERT in neurons with oxygen-glucose deprivation," Neuroscience, vol. 252, pp. 346-358, 2013.

[23] J. Zhao, Y. Zhao, W. Zheng, Y. Lu, G. Feng, and S. Yu, "Neuroprotective effect of curcumin on transient focal cerebral ischemia in rats," Brain Research, vol. 1229, pp. 224-232, 2008.

[24] A. Ricobaraza, M. Cuadrado-Tejedor, A. Pérez-Mediavilla, D. Frechilla, J. Del Río, and A. García-Osta, "Phenylbutyrate ameliorates cognitive deficit and reduces tau pathology in an alzheimer's disease mouse model," Neuropsychopharmacology, vol. 34, no. 7, pp. 1721-1732, 2009.

[25] J. K. Hyeon, M. Rowe, M. Ren, J.-S. Hong, P.-S. Chen, and D.-M. Chuang, "Histone deacetylase inhibitors exhibit antiinflammatory and neuroprotective effects in a rat permanent ischemic model of stroke: multiple mechanisms of action," The Journal of Pharmacology and Experimental Therapeutics, vol. 321, no. 3, pp. 892-901, 2007.

[26] H.-S. Suh, S. Choi, P. Khattar, N. Choi, and S. C. Lee, "Histone deacetylase inhibitors suppress the expression of inflammatory and innate immune response genes in human microglia and astrocytes," Journal of Neuroimmune Pharmacology, vol. 5, no. 4, pp. 521-532, 2010.

[27] M. Ying, R. Xu, X. Wu et al., "Sodium butyrate ameliorates histone hypoacetylation and neurodegenerative phenotypes in a mouse model for DRPLA," The Journal of Biological Chemistry, vol. 281, no. 18, pp. 12580-12586, 2006.

[28] S. I. Deutsch, R. B. Rosse, K. D. Long, B. L. Gaskins, J. A. Burket, and J. Mastropaolo, "Sodium butyrate, an epigenetic interventional strategy, attenuates a stress-induced alteration of MK-801's pharmacologic action," European Neuropsychopharmacology, vol. 18, no. 8, pp. 565-568, 2008.

[29] J. Rodrigo, A. P. Fernández, J. Serrano, M. A. Peinado, and A. Martínez, "The role of free radicals in cerebral hypoxia and ischemia," Free Radical Biology \& Medicine, vol. 39, no. 1, pp. 26-50, 2005.

[30] J. Yamada, S. Yoshimura, H. Yamakawa et al., "Cell permeable ROS scavengers, Tiron and Tempol, rescue PC12 cell death caused by pyrogallol or hypoxia/reoxygenation," Neuroscience Research, vol. 45, no. 1, pp. 1-8, 2003.

[31] Y. Li, Y. Bao, B. Jiang et al., "Catalpol protects primary cultured astrocytes from in vitro ischemia-induced damage," International Journal of Developmental Neuroscience, vol. 26, no. 3-4, pp. 309-317, 2008.

[32] P. Gonçalves, I. Gregório, T. A. Catarino, and F. Martel, "The effect of oxidative stress upon the intestinal epithelial uptake of butyrate," European Journal of Pharmacology, vol. 699, no. 1-3, pp. 88-100, 2013.

[33] B. R. S. Broughton, D. C. Reutens, and C. G. Sobey, "Apoptotic mechanisms after cerebral ischemia," Stroke, vol. 40, no. 5, pp. e331-e339, 2009.

[34] A. Vakili, S. Sharifat, M. M. Akhavan, and A. R. Bandegi, "Effect of lavender oil (Lavandula angustifolia) on cerebral edema and its possible mechanisms in an experimental model of stroke," Brain Research, vol. 1548, pp. 56-62, 2014.

[35] Y. Béjot, A. Prigent-Tessier, C. Cachia et al., “Time-dependent contribution of non neuronal cells to BDNF production after ischemic stroke in rats," Neurochemistry International, vol. 58, no. 1, pp. 102-111, 2011.

[36] G. Song, G. Ouyang, and S. Bao, "The activation of Akt/PKB signaling pathway and cell survival," Journal of Cellular and Molecular Medicine, vol. 9, no. 1, pp. 59-71, 2005.

[37] G. Bozlu, A. Atici, A. H. Turhan et al., "Platelet-activating factor antagonist (ABT-491) decreases neuronal apoptosis in neonatal rat model of hypoxic ischemic brain injury," Brain Research, vol. 1143, pp. 193-198, 2007.

[38] Y. Zhou, T. Lekic, N. Fathali et al., "Isoflurane posttreatment reduces neonatal hypoxic-ischemic brain injury in rats by the sphingosine-1-phosphate/phosphatidylinositol-3kinase/akt pathway," Stroke, vol. 41, no. 7, pp. 1521-1527, 2010. 

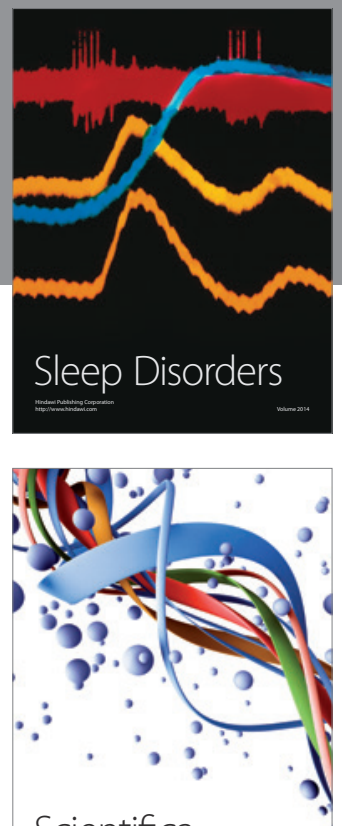

Scientifica
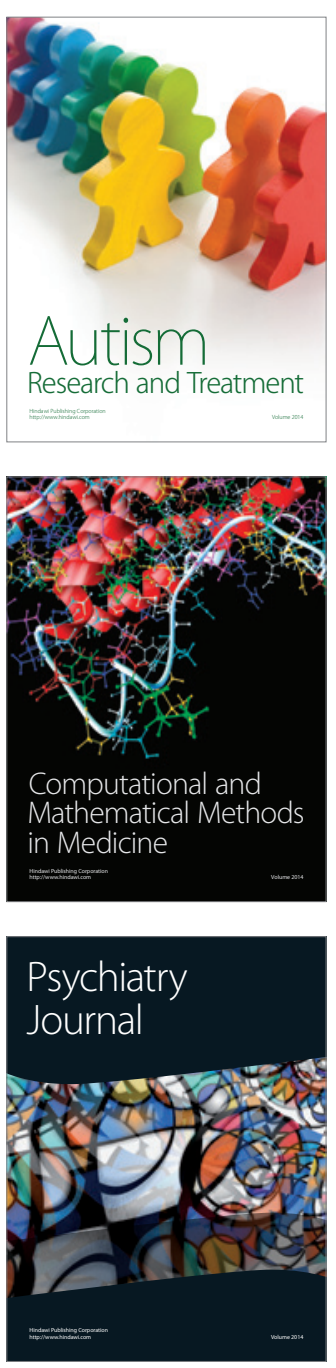
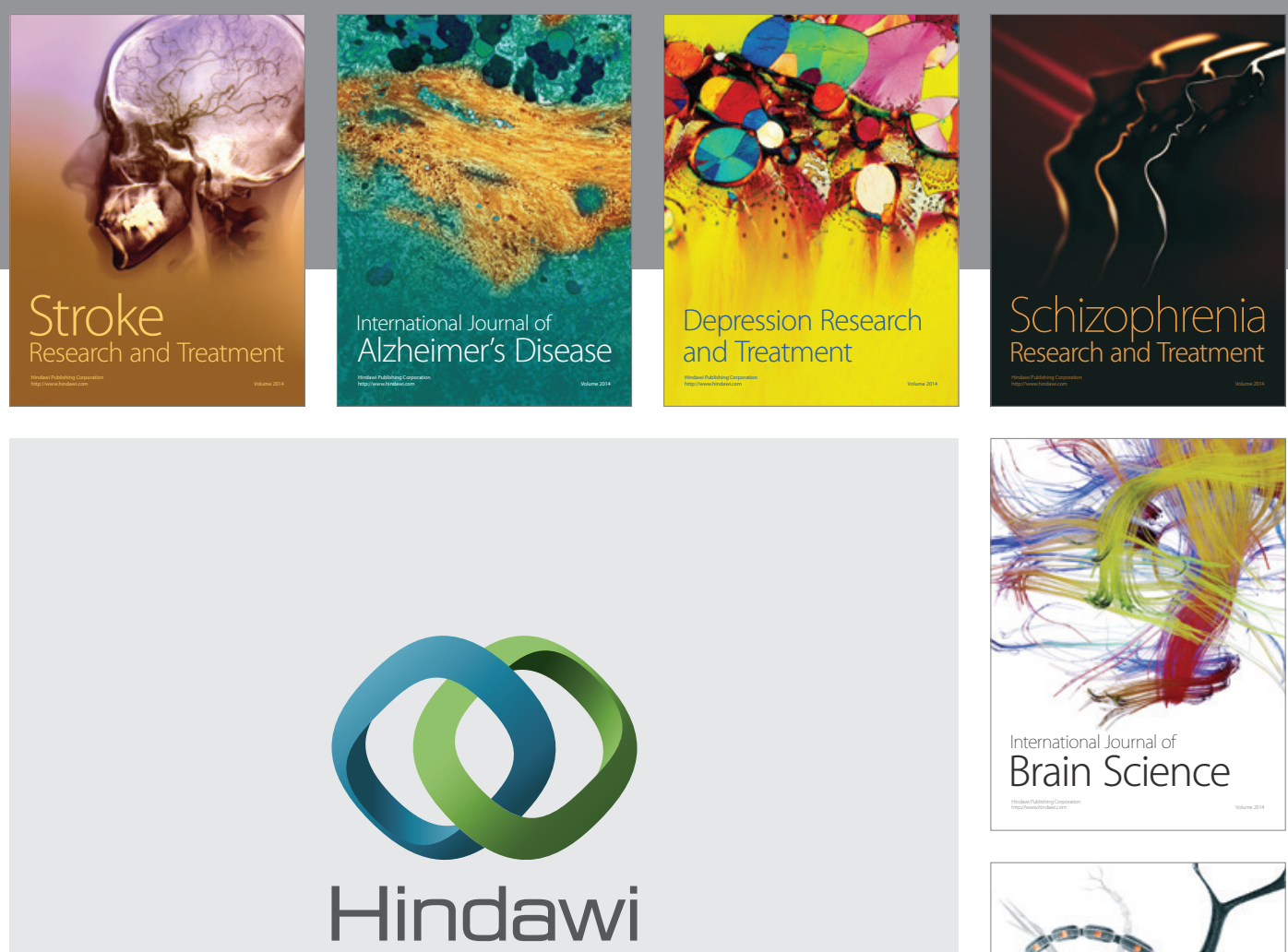

Submit your manuscripts at

http://www.hindawi.com
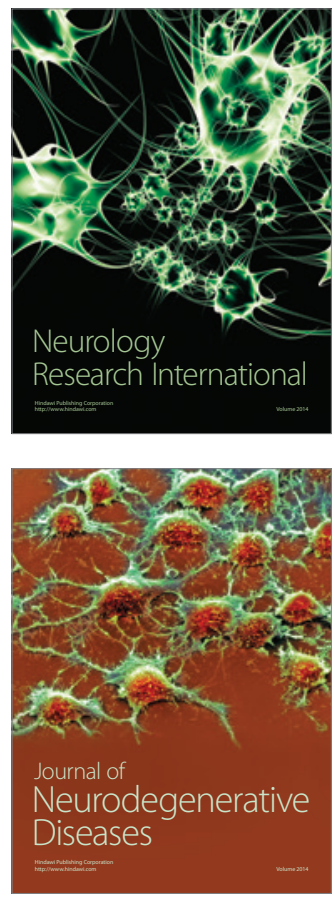

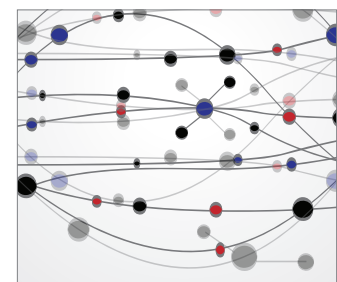

The Scientific World Journal
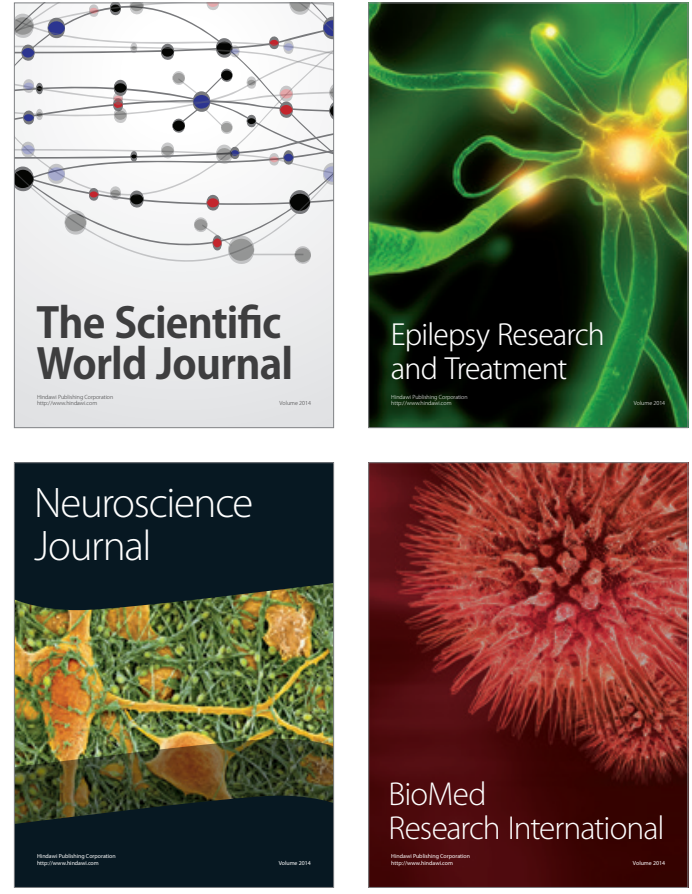

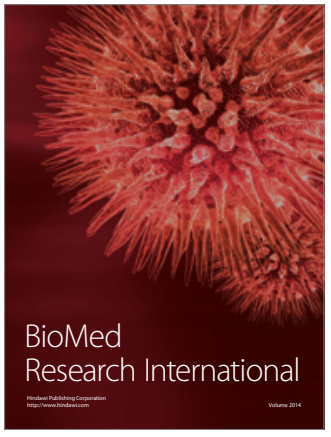

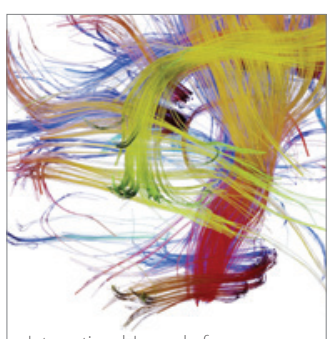

Brain Science

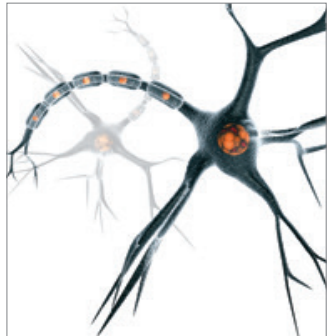

Neural Plasticity
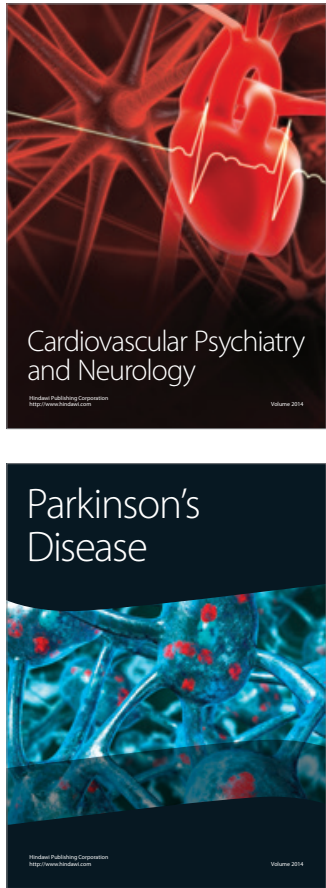